\title{
L'implant cochléaire: de la surdité au monde des entendants
}

\author{
Adrian Dalbert ${ }^{a}$, Dorothe Veraguth ${ }^{b}$, Alexander Huber $^{c}$ \\ ${ }^{a}$ Dr, Hôpital universitaire de Zurich; ${ }^{b}$ KD Dr, Hôpital universitaire de Zurich; ${ }^{c}$ Prof., Hôpital universitaire de Zurich
}

Une déficience auditive sévère ou une surdité totale est une maladie avec de lourdes conséquences pour les personnes concernées, qu'il s'agisse d'enfants ou d'adultes. L'implant cochléaire peut leur permettre d'accéder au monde des entendants ou de le réintégrer, avec un bénéfice individuel incontestable, mais aussi un avantage social important.

\section{Les troubles auditifs et leurs conséquences}

«La cécité sépare des objets, la surdité sépare des personnes.» Cette phrase de Helen Keller, écrivaine américaine sourde et aveugle, souligne de façon saisissante l'isolement extrême que la surdité crée chez les enfants et les adultes. Un à trois enfants sur mille naissent sourds et un sur mille perd l'audition avant d'atteindre l'âge adulte. Un tiers environ des plus de 65 ans souffre d'une surdité de l'oreille interne nécessitant un traitement - pourcentage qui augmente avec l'âge.

En matière de perte auditive, on distingue la surdité de transmission due généralement à des troubles de l'oreille moyenne, et la surdité de l'oreille interne. Alors que diverses possibilités existent pour traiter les cas de surdité de transmission (par ex. tubes tympaniques, prothèses de l'oreille interne, implants auditifs à ancrage osseux), les appareils auditifs sont la seule option pour les cas de surdité légère à moyenne de l'oreille interne. Toutefois, cette possibilité ne s'applique pas aux cas sévères de déficience auditive de l'oreille interne ou de surdité totale. Malgré les appareils auditifs, ces personnes restent très limitées dans leur capacité de compréhension et doivent souvent lire sur les lèvres ou recourir au langage des signes. Une communication orale est de plus en plus difficile. Chez les enfants sourds de naissance, le développement du langage est pratiquement inexistant. Les personnes souffrant d'une déficience auditive sévère ou d'une surdité post-linguale se retirent souvent du monde des entendants, avec pour conséquences un isolement social, des troubles psychiques et un cursus scolaire ou professionnel fortement impacté.

\section{L'implant cochléaire: la seule prothèse d'«organe sensoriel"}

Pour les personnes concernées, l'implant cochléaire est le seul moyen de réintégrer le monde de l'audition. Cette technique consiste à stimuler directement le nerf auditif par impulsions électriques en implantant une électrode dans la cochlée, ce qui nécessite une intervention de 90 minutes environ. L'implant cochléaire est à ce jour la seule prothèse susceptible de remplacer un organe sensoriel.

Les premiers implants cochléaires ont eu lieu il y a 40 ans et leur nombre n'a cessé d'augmenter depuis le début des années 90. En 1996, 42 patients ont bénéficié en Suisse d'un implant cochléaire, en 2016, ils étaient 243. Le nombre des patients bénéficiaires de ce traitement a donc été multiplié par six au cours de cette période.

L'implant cochléaire redonne à une grande majorité des patients une capacité de compréhension qui leur permet de mener une vie professionnelle normale. Chez les enfants nés sourds, il est recommandé de réaliser

Le nombre de patients ayant bénéficié d'un tel implant a presque été multiplié par six au cours des 20 dernières années.

l'implantation dans les deux premières années afin de favoriser un bon développement du langage oral et une scolarisation normale. Pour atteindre cet objectif et détecter le plus tôt possible les enfants atteints d'une perte auditive ou de surdité, le dépistage auditif a été introduit en 1999 dans toute la Suisse. Ce dépistage, tout comme l'implant cochléaire, est une mesure qui occasionne des coûts de santé, mais qui, sur le long 
terme, permet à la société d'économiser les coûts d'enseignement spécialisé, les réorientations, les rentes et autres prestations de soutien - sans parler des bénéfices directs pour les implantés. Les deux exemples suivants permettront de l'illustrer.

\section{Cavalière passionnée et banquière grâce à un implant cochléaire}

T. W. est née l'été 1998 au terme d'une grossesse sans problème. Il n'y avait aucun antécédent de déficience auditive dans sa famille. Ses parents ont toutefois remarqué que $\mathrm{T}$. W. ne réagissait qu'aux stimulations visuelles et qu'elle restait totalement indifférente aux bruits, même les plus forts. Des examens plus approfondis ont donc été effectués, dont une audiométrie du tronc cérébral à un an et demi. Le diagnostic a confirmé les soupçons des parents d'une surdité sévère des deux côtés.

Les appareils auditifs utilisés en premier n'ayant pas permis à T. W. d'entendre suffisamment pour le développement du langage oral, la possibilité d'un implant cochléaire a été exposée aux parents et réalisée à l'âge de deux ans.

Aujourd'hui, T. W. a 19 ans et travaille dans une banque. Après l'implant cochléaire, elle a réussi à rattraper son retard dans le développement du langage. Elle a suivi un cursus scolaire normal avec le soutien du service audio-pédagogique et a achevé l'école secondaire ainsi que son apprentissage avec succès.

En dehors de l'école et de son métier, T. W. est une jeune femme active, mais qui dépend de son implant cochléaire pour entendre et pratiquer entre autres son passe-temps préféré, l'équitation.

\section{Rester médecin grâce à un implant cochléaire}

M. P. a pris pour la première fois conscience de sa déficience auditive à l'âge de 30 ans. Son métier de médecin exigeant une bonne communication avec ses patients et ses collaborateurs, il a porté des appareils auditifs. Comme son audition continuait à se détériorer, réduisant sa capacité à communiquer malgré les appareils auditifs, M.P. a opté pour un implant cochléaire, d'abord unilatéral. La nette amélioration apportée par l'implant et la détérioration continue de sa capacité auditive à l'autre oreille l'ont amené un peu plus tard à accepter une seconde implantation controlatérale.

M. P. est très satisfait du résultat. Ce médecin cadre anesthésiste, appelé à intervenir en salle de réanimation ou d'opération, souvent en situation d'urgence, ne rencontre aucun problème dans son environnement de travail. Ce quotidien professionnel n'aurait pas été envisageable sans l'implant cochléaire.

\section{Résumé}

L'implant cochléaire permet de traiter avec succès les patients souffrant de déficience auditive sévère ou de surdité totale, et de favoriser ou rétablir la communication orale. Le nombre de patients ayant bénéficié d'un tel implant a presque été multiplié par six au cours des 20 dernières années. En plus du bénéfice personnel évident, cette méthode présente également une utilité sociale: les enfants qui sont traités à temps ont de bonnes chances de développer le langage oral et de suivre une scolarité normale, et les adultes concernés peuvent poursuivre leur activité professionnelle. Cela n'était pas possible auparavant et ne le serait toujours pas aujourd'hui, dans la plupart des cas, sans l'implant cochléaire. Il en résulterait une grande perte en termes de potentiel individuel non exploité et, à plus longue échéance, en termes de coûts pour la société. En effet, les coûts de santé d'environ 50000 francs engendrés par l'implant doivent être mis en relation avec des économies bien plus importantes réalisées sur la durée, comme les coûts de l'enseignement spécialisé, les réorientations, les rentes et autres prestations de soutien, devenus superflus. 